\title{
Combination of WAGR and Potocki-Shaffer contiguous deletion syndromes in a patient with an 11p11.2-p14 deletion
}

Dominique Brémond-Gignac ${ }^{1,2}$, John A Crolla ${ }^{3}$, Henri Copin ${ }^{4}$, Agnès Guichet ${ }^{5}$, Dominique Bonneau $^{5}$, Laurence Taine ${ }^{6}$, Didier Lacombe ${ }^{6}$, Clarisse Baumann ${ }^{7}$, Brigitte Benzacken ${ }^{8}$ and Alain Verloes*,2,7

\footnotetext{
${ }^{1}$ Department of Ophthalmology, Robert Debré Hospital, AP-HP, Paris, France; ${ }^{2}$ Unité INSERM E676, Paris, France; ${ }^{3}$ Wessex Regional Genetics Laboratory, Salisbury District Hospital, Salisbury, UK; ${ }^{4}$ Department of Cytogenetics, Lariboisière Hospital, AP-HP, Paris, France; ${ }^{5}$ Department of Genetics, University hospital, Angers, France; ${ }^{6}$ Department of Genetics, Pellegrin University Hospital, Bordeaux, France; ${ }^{7}$ Department of Genetics, Robert Debré Hospital, Paris, France; ${ }^{8}$ Department of Histo-Embryo-Cytogenetics, Jean Verdier Hospital, Paris, France
}

Aniridia, Wilms tumor, genitourinary abnormalities, growth and mental retardation are the cardinal features of the WAGR 11p13 deletion syndrome. The Potocki-Schaffer syndrome or proximal 11p deletion syndrome (previously DEFECT11 syndrome) is a contiguous gene syndrome associated with deletions in 11p11.2, principal features of which are multiple exostoses and enlarged parietal foramina. Mental handicap, facial dysmorphism and craniosynostosis may also be associated. We report a patient with combined WAGR and Potocki-Shaffer syndromes, and obesity. She presented with aniridia, cataract, nystagmus, corneal ulcers and bilateral congenital ptosis. A left nephroblastoma was detected at 15 months. Other features included moderate developmental delay, growth deficiency, facial dysmorphism, multiple exostoses and cranial lacunae. High-resolution and molecular cytogenetics confirmed a del(11)(p11.2p14.1) deletion with a proximal breakpoint between the cosmid D08153 and the BAC RP11$104 \mathrm{M} 24$ to a distal breakpoint between cosmids C08160 (D11S151) and F1238 (D11S1446). The deletion therefore includes EXT2, ALX4,WT1 and PAX6. This case appears to be the second patient reported with this combined deletion syndrome and confirms the association of obesity in the WAGR spectrum, a feature previously reported in four cases, and for which the acronym WAGRO has been suggested. Molecular and follow-up data on the original WAGRO case are briefly presented.

European Journal of Human Genetics (2005) 13, 409-413. doi:10.1038/sj.ejhg.5201358

Published online 26 January 2005

Keywords: WAGR; WAGRO; exostosis; Potocki-Schaffer syndrome; contiguous gene deletion syndrome; obesity

Introduction

WAGR syndrome is a rare contiguous gene disorder characterized by Wilms tumor, aniridia, genitourinary

*Correspondence: Professor A Verloes, Clinical Genetics Unit, Genetic Department, Robert Debré Hospital, 48, Boulevard Sérurier, Paris 75019, France. Tel: + 3314003 5342; Fax: + 3314003 5344;

E-mail: alain.verloes@rdb.ap-hop-paris.fr

Received 8 June 2004; revised 1 October 2004; accepted 6 October 2004 abnormalities, growth and mental retardation. This syndrome is caused by hemizygosity for the PAX6, WT1 and other genes, and is the result of visible or cryptic deletions, all of which invariably involve part or all of chromosome band 11p13. Proximal 11p deletion syndrome (PotockiSchaffer syndrome or, previously, DEFECT11 syndrome) is a contiguous gene syndrome associated with deletions in the $11 \mathrm{p} 11-\mathrm{p} 12$ region. The principal clinical features are multiple exostoses and enlarged parietal foramina, 
although mental handicap, craniosynostosis and facial dysmorphism may also be associated. The main features of Potocki-Shaffer syndrome have been reported to be related to deletions of EXT2 causing multiple exostoses ${ }^{1}$ and ALX4 causing enlarged parietal foramina. ${ }^{2}$ We describe a patient with a contiguous gene deletion syndrome that encompasses both proximal 11p and WAGR deletion regions of $11 \mathrm{p}$.

\section{Clinical report \\ Patient report}

Patient 1 A 25-year-old girl with WAGR syndrome was referred to the ophthalmology clinic for a visual evaluation. Ptosis was present since birth and aniridia was diagnosed at 3 months. A left kidney tumor was detected at 15 months, surgically removed and followed by chemotherapy. Cataract surgery was performed at the age of 7 and 12 years. At 6 years, multiple exostoses of long bones were found during a radiological check-up. Puberty occurred at age 10. Mammary hypertrophy was surgically treated and severe obesity appeared at age 17 years.

When examined at age 25 , the patient was $150 \mathrm{~cm}$ tall $(-2.4 \mathrm{SD})$, weighted $95 \mathrm{~kg}(+7 \mathrm{SD})$ and had an OFC of $55 \mathrm{~cm}$ (median). Her BMI was 42. Obesity was predominantly truncal. She had facial dysmorphism (Figure 1a), bilateral ptosis, downslanted palpebral fissures, flat malar areas, low set ears, patulous lips, micrognathia and crowded teeth. Facial hair extended to the mandibular angle (Figure 1b). At ophthalmologic examination, horizontal nystagmus, bilateral aphakia, complete bilateral aniridia with neovascularization, scars of corneal ulcer, diffuse corneal opacities preventing examination of the fundi and ocular hypertension were found. She had residual visual acuity that allowed her to deambulate. Hypertension and proteinuria were present. Renal function was otherwise normal. She had mild to moderate mental retardation associated with mood disturbances, obsessivecompulsive behavior, hyperphagia, temper tantrums and intolerance to frustration, requiring antipsychotic medications. Basic screening for diabetes was negative.

Skeletal survey showed multiple, blunt-shaped exostoses on long bones and vertebrae (Figure $2 \mathrm{a}$ and b). Small foramina were visible on frontal X-ray of the skull (Figure 2c), but were not visible on profile view. These foramina were indeed localized in the occipital bone rather than parietal bone.

Patient 2 This patient was originally reported by Marlin et $\mathrm{al}^{3}$ as WAGRO syndrome. She had aniridia, external genital hypoplasia and severe, early-onset obesity (up to +10 SD). Cytogenetic investigations had shown a 11p12p14 deletion (Figure 3). At the last follow-up at age 14 , this girl was $155 \mathrm{~cm}$ tall $(-0.6 \mathrm{SD})$ and weighted $65 \mathrm{~kg}$ $(+2.5 \mathrm{SD})$, corresponding to a BMI of 26 . OFC was $57.5 \mathrm{~cm}$
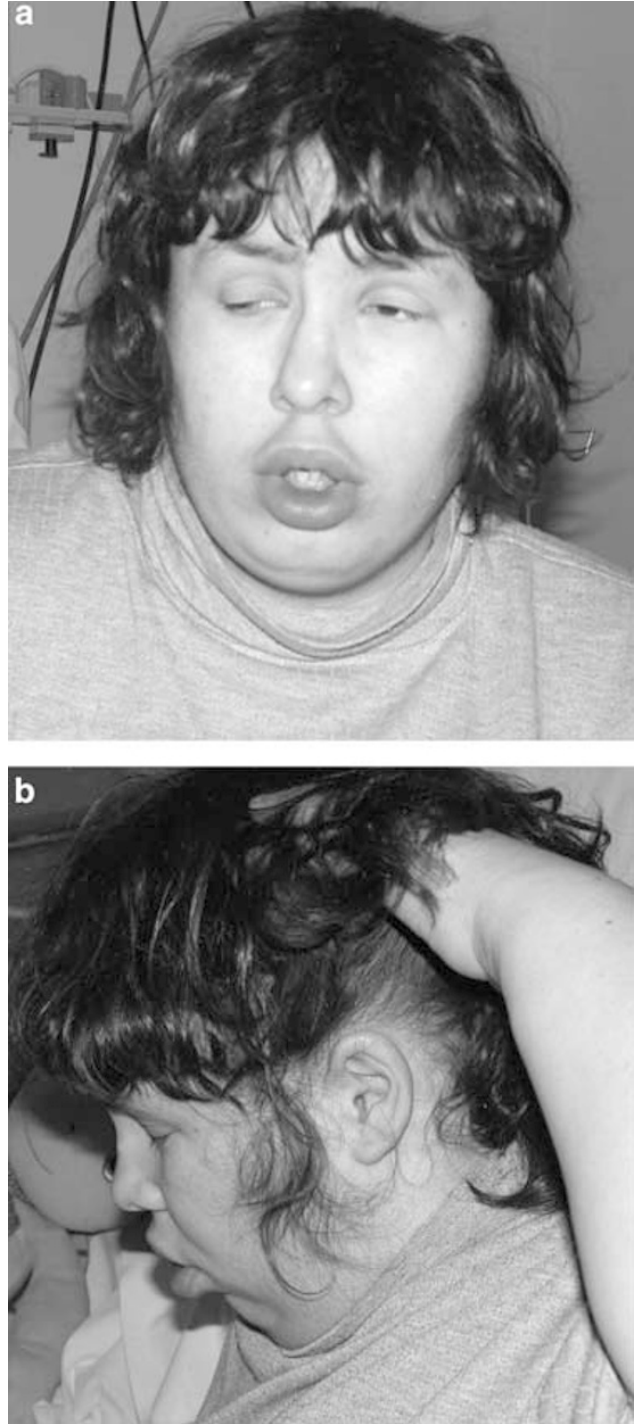

Figure 1 Patient 1: (a) face and (b) profile view of patient 1. Note facial dysmorphism, unusual hairline on the temporal area and obesity.

(2.1SD). She had gynoid obesity. She was legally blind, with only light perception. Onset of obesity was in infancy. Despite hyperphagia, weight was stabilized through strict dietary and psychological management, allowing BMI to drop to the 'overweight range'. She had moderate to severe mental retardation, and pervasive developmental disorder with chronic anxiety, poor acceptance of change, logorrhea, echolaly, poor social interactions, labile mood, and bouts of aggressiveness and motor agitation that required treatment with risperidone. She was not able to wash or to dress herself. Owing to communication difficulties, formal IQ was not possible. 

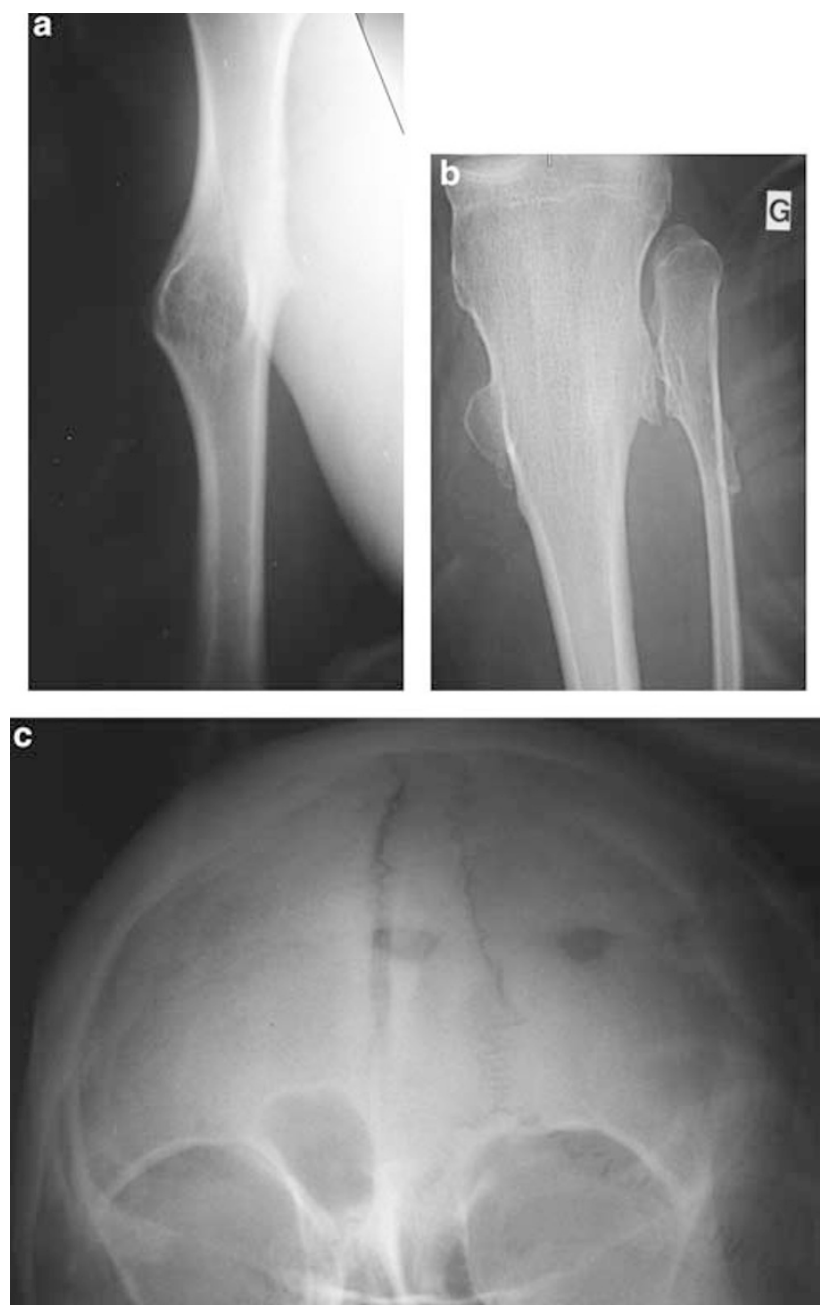

Figure 2 Skeletal survey: bony exostoses (a) of the humerus and (b) upper tibia and fibula and (c) small cranial foramina.

\section{Cytogenetic investigations}

Patient 1 Cytogenetic and fluorescence in situ hybridization (FISH) studies were carried out on PHA-stimulated peripheral blood metaphases. Conventional high-resolution R-banding found a deletion in the proximal short arm of one 11 homologue and the breakpoints were described as: $\operatorname{del}(11)(\mathrm{p} 11.2 \mathrm{p} 14.1)$. FISH was subsequently carried out on metaphases from fixed cells using the following probes (distal to proximal) F1238 (D11S1446), CO8160 (D11S151), AO4160 (D11S914), FO2121, FAT5 (PAX6), B2.1 (WT1), c111-458 (D11S1776), c1-11-474 (D11S1103), RP11-104M24 (EXT2), RP11-582N4 (ALX4) and DO8153 (RAPSYN). The probes FO2121, FAT5 (PAX6 exons 1-4), cB2.1 (WT1) and F02121 map to the WAGR critical region with the RP-11 BACs 582N4 and 104M24 (http://www.ensembl.org/ Homo_sapiens/cytoview) marking the Potocki-Schaffer critical region. The map positions of the probes used can

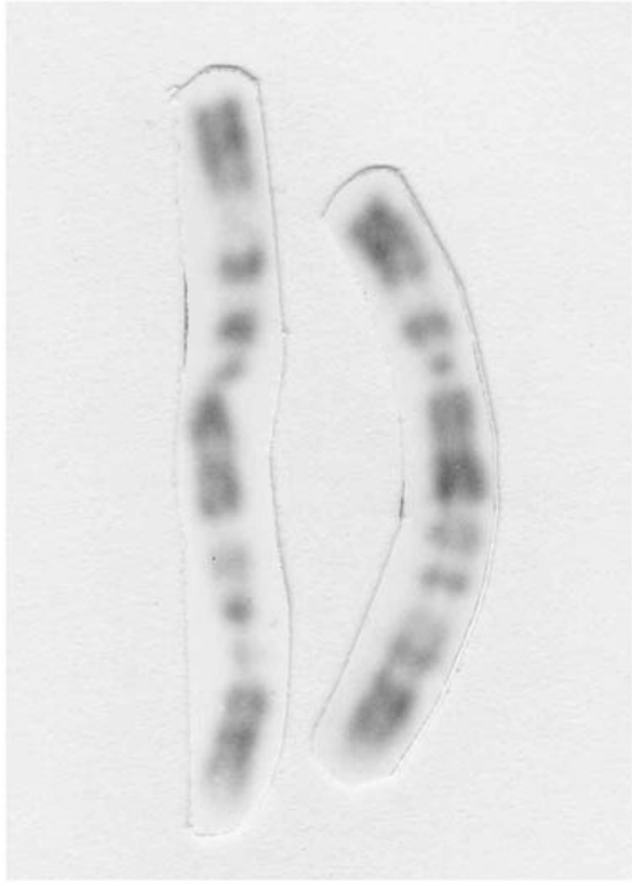

Figure 3 Partial high-resolution karyotype of patient 2 illustrating the large $11 \mathrm{p} 12 \mathrm{p} 14.3$ deletion on the left chromosome.

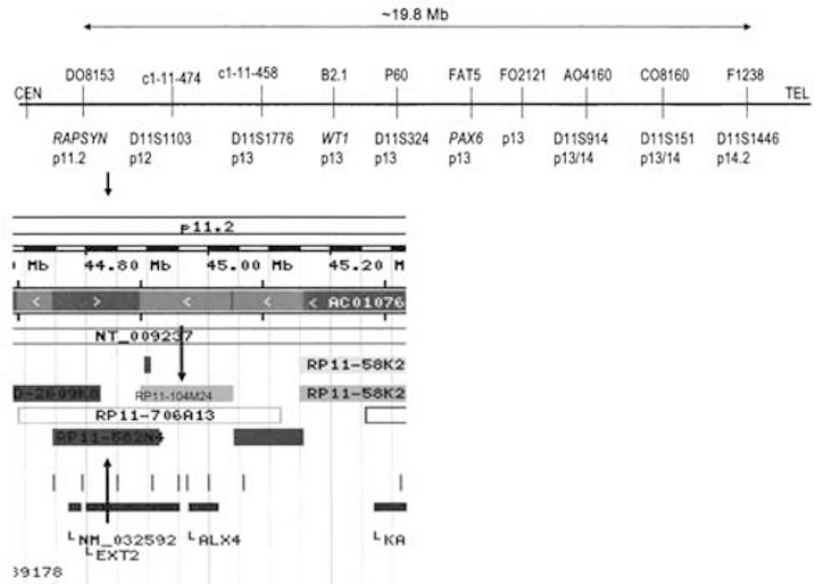

Figure 4 A map of the $11 p$ region (not drawn to scale) showing the location of the probes used in the FISH analysis of patient 1 . The inset is taken from Ensembl shows the positions of the two RP11 BACs used mapping to EXT2 and ALX4, respectively.

be seen in Crolla and van Heyningen ${ }^{4}$ and in Figure 4 . Between 7 and 10 metaphases were examined after FISH with each probe. On the basis of the FISH results, it was possible to redefine the cytogenetic breakpoints to the following: del(11)(p11.2p14). 
The deletion was shown to span from a point located proximally between DO8153 (present) and 104M24 (deleted) on $11 \mathrm{p} 11.2$ to the interval between D11S151 in 11p13-14 (deleted) and D11S1446 in 11p14.2 (not deleted) Based on the Ensembl Cytoview data, the size of the deletion was thus between 14.2 and $19.8 \mathrm{Mb}$ (Figure 3).

Patient 2 Only partial FISH study was possible. It confirmed deletion of PAX6 and WT1. The proximal breakpoint fall between BACs RP11-1G18 (11p12 - deleted) and RP11-12C11 (11p11.2 - not deleted). A deletion of PDE3B gene located in 11p15 was excluded by specific FISH with the RP11-699C18 BAC. The deleted region spanned approximately $20 \mathrm{Mb}$, based on Ensembl databank.

\section{Discussion}

WAGR is an acronym for: Wilms tumor, Aniridia, Genitourinary anomalies/gonadoblastoma and Mental Retardation. A combination of two or more of these clinical features is normally required for an individual to be diagnosed with WAGR syndrome. The feature invariably present in all documented cases is aniridia. WAGR was clinically delineated by Miller et al. ${ }^{5}$ Its cytogenetic basis, a deletion of $11 \mathrm{p} 13$ was recognized by Riccardi et al. ${ }^{6}$ Urogenital and renal abnormalities and predisposition to nephroblastoma are related to hemizygosity for the Wilms tumor suppressor gene WT1. Ocular defects result from hemizygosity for the PAX6 gene and although several genes have been implicated as the cause of neurological defects, no candidate gene(s) have so far been identified.

The coexistence of WAGR and obesity was first reported by Marlin in a 6-year-old girl with $\operatorname{del}(11)(\mathrm{p} 12 \mathrm{p} 14) .{ }^{3}$ This combination phenotype was confirmed in two further reports. ${ }^{7,8}$ However, the breakpoints of the deletion were not determined with molecular tools in previously published cases. Marlin proposed the acronym WAGRO for the association of WAGR and obesity. Our patient 1 has bona fide WAGRO syndrome. The frequency of obesity in WAGR syndrome is not established, as many case reports deal with younger children or focus only on renal and ophthalmological defects. Interestingly, in case 1 , the onset of obesity was delayed till the end of puberty, indicating that this feature could be more common than usually thought. Marlin suggested that an 'obesity gene' could be present in the deletion. $P D E 3 B$, a gene involved in a genetic form of murine obesity, and the only candidate gene known in the vicinity of the WAGR region, was mapped distal to the deletion, even in patient 2 . At this point, the etiology and pathogeny of obesity remains unsolved in WAGRO. Comparison of the deletions in adult patients with WAGR or WAGRO should be performed to address whether it results from a single gene effect, or is a more general and variable expression of the general mental and behavioral status.
Potocki-Shaffer syndrome (also known as 'Proximal 11p Deletion syndrome', 'P11pDS' and previously 'DEFECT11 syndrome'), a contiguous gene disorder presenting with parietal foramina, microbrachycephaly and variable mental retardation in association with $\operatorname{del}(11)(\mathrm{p} 11.12 \mathrm{p} 12)$, was first reported in $1993 .{ }^{9}$ Subsequently, Potocki and Shaffer ${ }^{10}$ and Bartsch and co-workers ${ }^{1}$ fully delineated this contiguous gene syndrome in several patients, including familial cases. The main clinical features are multiple exostoses, enlarged parietal foramina, craniofacial dysmorphism reminiscent of Saethre-Chotzen syndrome and mental retardation. Hemizygosity for EXT2 explains the exostoses $^{11}$ and hemizygosity for $A L X 4$ is responsible for parietal dysostosis. ${ }^{2,12}$ Hall et al ${ }^{13}$ reported a multigenerational family with multiple exostoses and parietal foramina but no mental retardation or craniofacial abnormalities. They showed that the EXT2 gene and the ALX4 gene were deleted, indicating that mental retardation and craniofacial abnormalities resulted from deletions outside the very narrow D11S1785-D11S1385 interval. An update on phenotype/genotype relationships was recently given in this journal. ${ }^{14}$

To our best knowledge, only one case has been previously reported with the combination of WAGR and Potocki-Shaffer phenotypes, ${ }^{15}$ and a del(11)(p11.2p14). Partial molecular characterization of the deletion were subsequently published, $^{16}$ confirming the cytogenetic breakpoints. A deletion of ALX4 was not specifically mentioned. Detailed characterization by a combination of cytogenetic, molecular cytogenetic and other molecular technologies of cases with interstitial 11p deletions will help to further define genotype/karyotype correlations and may eventually identify candidate genes for obesity and mental retardation in this region of the genome.

\section{References}

1 Bartsch O, Wuyts W, Van Hul W et al: Delineation of a contiguous gene syndrome with multiple exostoses, enlarged parietal foramina, craniofacial dysostosis, and mental retardation, caused by deletions in the short arm of chromosome 11. Am J Hum Genet 1996; 58: 734-742.

2 Wu YQ, Badano JL, McCaskill C, Vogel H, Potocki L, Shaffer LG: Haploinsufficiency of ALX4 as a potential cause of parietal foramina in the $11 \mathrm{p} 11.2$ contiguous gene-deletion syndrome. Am J Hum Genet 2000; 67: 1327-1332.

3 Marlin S, Couet D, Lacombe D, Cessans C, Bonneau D: Obesity: a new feature of WAGR (del 11p) syndrome. Clin Dysmorphol 1994; 3: $255-257$

4 Crolla JA, van Heyningen V: Frequent chromosome aberrations revealed by molecular cytogenetic studies in patients with aniridia. Am J Hum Genet 2002; 71: 1138-1149.

5 Miller RW, Fraumeni Jr JF, Manning MD: Association of Wilms's tumor with aniridia, hemihypertrophy and other congenital malformations. N Engl J Med 1964; 270: 922-927.

6 Riccardi VM, Sujansky E, Smith AC, Francke U: Chromosomal imbalance in the Aniridia-Wilms' tumor association: 11p interstitial deletion. Pediatrics 1978; 61: 604-610.

7 Tiberio G, Digilio MC, Giannotti A: Obesity and WAGR syndrome. Clin Dysmorphol 2000; 9: 63-64. 
8 Gul D, Ogur G, Tunca Y, Ozcan O: Third case of WAGR syndrome with severe obesity and constitutional deletion of chromosome (11)(p12p14). Am J Med Genet 2002; 107: 70-71.

9 Shaffer LG, Hecht JT, Ledbetter DH, Greenberg F: Familial interstitial deletion $11(\mathrm{p} 11.12 \mathrm{p} 12)$ associated with parietal foramina, brachymicrocephaly, and mental retardation. Am J Med Genet 1993; 45: 581-583.

10 Potocki L, Shaffer LG: Interstitial deletion of 11(p11.2p12): a newly described contiguous gene deletion syndrome involving the gene for hereditary multiple exostoses (EXT2). Am J Med Genet 1996; 62: 319-325.

11 Stickens D, Clines G, Burbee D et al: The EXT2 multiple exostoses gene defines a family of putative tumour suppressor genes. Nat Genet 1996; 14: 25-32.
12 Mavrogiannis LA, Antonopoulou I, Baxova A et al: Haploinsufficiency of the human homeobox gene ALX4 causes skull ossification defects. Nat Genet 2001; 27: 17-18.

13 Hall CM, Washbrook J: Radiological Electronic Atlas of Malformation Syndromes. Oxford: Oxford Electronic Publishing, 2000.

14 Wuyts W, Waeber G, Meinecke P et al: Proximal 11p deletion syndrome (P11pDS): additional evaluation of the clinical and molecular aspects. Eur J Hum Genet 2004; 12: 400-406.

15 McGaughran JM, Ward HB, Evans DG: WAGR syndrome and multiple exostoses in a patient with $\operatorname{del}(11)(\mathrm{p} 11.2 \mathrm{p} 14.2) . J$ Med Genet 1995; 32: 823-824.

16 Wuyts W, Van Hul W, Wauters J et al: Positional cloning of a gene involved in hereditary multiple exostoses. Hum Mol Genet 1996; 5: $1547-1557$. 